\title{
A case of shingles (herpes zoster) post hair transplant
}

Paul Cotterill, MD Toronto, Ontario, Canada pau@drcotterill.com

A 48-year-old healthy male, with no allergies, taking Propecia ${ }^{\circledR}$, had his second transplant in January of 2011. Past history was unremarkable.

The transplant was to thicken the frontal portion of the scalp including the hairline. Approximately 2,200 follicular unit grafts were transplanted. The procedure was uneventful.

The patient was called the evening after surgery and was recovering nicely. The day after surgery all patients routinely return for a hair wash. At that point the scalp donor and recipient area were all healing well. The patient remarked that his left eye hurt a little bit, but on examination of the eye and surrounding skin, all looked fine. There was no redness to the eye or skin and nothing atypical was seen. The next day he called and said his eye felt gritty after getting out of the tub while washing his hair and scalp, and he thought he saw a small rash above the left eyebrow, but well down on the forehead and well below the transplant. I said to go more lightly on the icing and to call or see me the next day if it had not improved.

The patient returned the next day as he mentioned that overnight he had developed some pimples around his nose. When I saw him, his left eye was slightly pinkish, still gritty, and now painful with a rash over the eyebrow and a painful nose. The pimples he described were actually clear fluid filled vesicles on the side of the nose. This is a big tip-off to shingles until proven otherwise. He was seen by an ophthalmologist within 2 hours.

Of note, the patient could not remember any history of chicken pox, cold sores, or any type of herpetic problems. He was placed on an antibiotic (Keflex), an anti-viral (Famvir), and a topical corticosteroid eye drop. The patient returned to my office at 7 days post-transplant for suture removal. Photos were taken at that time (Figures 1 and 2). At 5 days after the diagnosis of shingles and being on medication, there were more vesicles but they were beginning to resolve. The vesicles stopped just shy of the hairline; however, his

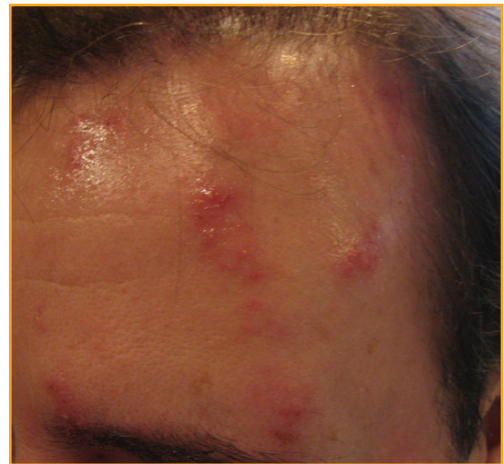

Figure 1. Patient 7 days post hair transplant with active facial herpes zoster.



Figure 2. Same patient showing ocular involvement.

eye continued to be pink and sore. At 6 weeks post-surgery, the skin involvement had completely cleared but the patient was still taking corticosteroid eye drops. At 15 months, the patient was continuing with the steroid drops and currently, at 20 months, the patient is still not able to be weaned off the

daily eye drops without a return of a watery, pink eye. There has been no permanent eye damage and the patient was told by his ophthalmologist that eventually the condition will burn itself out and drops will not be required. The transplants were completely unaffected by the shingles.

Very little has been reported in the literature regarding shingles and hair transplants. I have had 3 patients over the past 24 years who developed shingles near the transplant area within 2-3 days post-transplant. All 3 patients were in good health and in the other 2 prior cases the problem resolved itself with no permanent sequelae. It is important to be able to recognize shingles at an early stage and to initiate treatment quickly. Antiviral treatments, such as Famvir, can help to reduce the severity and duration of herpes zoster if a 7-10 day course of these drugs is started within 72 hours of the appearance of the characteristic rash. Uveitis (inflammation inside the eye) occurs in about $40 \%$ of patients with ocular shingles. If there is any suspicion of eye involvement, the patient should be quickly seen and followed by an ophthalmologist. Damage can occur to the iris. Elevated eye pressure may be present, which could lead to the development of glaucoma. ${ }^{1}$ In most patients, the uveitis seen in ocular shingles lasts just a few weeks, but in some patients, it may come and go for many years.

\section{Reference}

1. Shaikh, S., and C.N. Ta. Evaluation and management of herpes zoster ophthalimicus. Am Fam Physician. 2002(Nov 1); 66(9):1723-1730. http://www.aafp.org/afp/2002/1101/ p1723.html 